\title{
IMPORTÂNCIA DA ANÁLISE DE ÁGUA PARA A SAÚDE PÚBLICA: PESQUISA DE NITRATO EM ÁGUAS DE ABASTECIMENTO PÚBLICO E POÇOS SUBTERRÂNEOS
}

\author{
Bianca Sant'Ana Verly, Erika Kushikawa Saeki, Éderson da Silva Stelato \\ Centro de Laboratório Regional Instituto Adolfo Lutz de Presidente Prudente-V, Núcleo de Ciências Químicas e \\ Bromatológicas, Presidente Prudente-SP. E-mail: biancaverly@ial.sp.gov.br \\ Agência de fomento: Instituto Adolfo Lutz
}

\section{RESUMO}

A água para consumo humano deve apresentar características sanitárias e toxicológicas adequadas. Dentre os poluidores das águas, as altas concentrações de nitrato apresentam um grande risco para a saúde pública e animal. Este estudo objetivou avaliar a qualidade água quanto à concentração de íon nitrato em nitrogênio em amostras da rede pública de abastecimento e em poços particulares, através do método de espectrofotometria na região do ultravioleta, no período de março a junho de 2016. Foram analisadas 1026 amostras de água de abastecimento e 17 amostras de poços. Dentre as amostras analisadas da rede de abastecimento, todas estavam dentro do valor permitido pela legislação, já as amostras de poços particulares, 5 estavam acima do limite aceitável. Concluiu-se que apesar das amostras de abastecimento público estar dentro do aceitável, o monitoramento é sempre importante. Nas amostras de poços se faz necessária a conscientização de cada explorador sobre a qualidade da água.

Palavras-chave: água subterrânea, saúde pública, qualidade da água, vigilância sanitária, contaminação química.

\section{IMPORTANCE OF WATER ANALYSIS FOR PUBLIC HEALTH: NITRATE RESEARCH IN PUBLIC WATER SUPPLY AND GROUNDWATER}

\begin{abstract}
The water for human consumption must show health and toxicological characteristics appropriate. Among the polluters of water, high concentrations of nitrate present a great risk to public and animal health. The objective of this study was to evaluate the water quality for the concentration of nitrate ion in samples of public supply and private wells, by the spectrophotometric method in the ultraviolet region, from march to june, 2016. Were analyzed 1,026 public supply samples and 17 groundwater samples. Among the analyzed samples of public supply, all were within the amount allowed by law, however, 5 private groundwater samples were above the acceptable limit. The conclusion was that even the public supply samples are according to the legislation, monitoring is always important. In the sample wells is necessary awareness of each operator on the quality of water.
\end{abstract}

Keywords: groundwater, public health, water quality, health surveillance, chemical contamination.

\section{INTRODUÇÃO}

Estima-se que cerca de $70 \%$ da superfície do planeta Terra é constituído por água, onde $3 \%$ são de água doce, e desse total $98 \%$ é água subterrânea. A água disponibilizada para consumo que são destinadas ao abastecimento público é em torno de $10 \%$, 23\% para o setor da indústria e $67 \%$ para a agricultura. A disponibilidade desse recurso hídrico é preocupante, pois a quantidade de mananciais de água em condições de vazão e qualidade adequada para o abastecimento da população está cada vez mais reduzida ${ }^{1,2}$.

No Estado de São Paulo, a qualidade da água para o consumo humano é monitorada através de coletas de amostras mensais realizadas pelas Vigilâncias Sanitárias municipais, a partir de análises microbiológicas: pesquisa de coliformes totais e Escherichia coli; e físicoquímicos: $\mathrm{pH}$, cor, turbidez, cloro residual livre e 
fluoreto, estabelecidos pelo Programa de Vigilância da Qualidade da Água para Consumo Humano (PROAGUA) ${ }^{3}$.

Algumas substâncias nocivas para a saúde humana podem ser encontradas na água, como os compostos de nitrogênio em seus diferentes estágios de oxidação. $O$ nitrato é encontrado em baixas concentrações em águas superficiais, porém em águas subterrâneas pode alcançar quantidades acima do permitido pela legislação ${ }^{4}$. A Portaria $n^{\circ} 2.914$, de 12 de dezembro de 2011 do Ministério da Saúde estabelece o padrão de potabilidade da água para consumo humano no Brasil, no qual determina que seja de até $10 \mathrm{mg} / \mathrm{L}$ o teor máximo de íon nitrato em nitrogênio ${ }^{5}$.

A presença de íon nitrato em águas subterrâneas pode ocorrer principalmente por cultivo do solo, aplicação de fertilizantes orgânicos e sintéticos nitrogenados em plantações, sistemas de saneamento através de tanques sépticos ou fossas rudimentares, vazamentos de redes de esgotos, contato de rios contaminados com áreas de captação de poços ${ }^{6} \mathrm{e}$ a ação do necrochorume provenientes de cemitérios ${ }^{7}$. Além disso, a ingestão de nitrato pela população também pode ser através de carnes e embutidos, na forma de conservantes e corantes $^{8}$.

O íon nitrato quando acima do permitido pela legislação torna-se prejudicial à saúde porque pode estar relacionado com 0 desencadeamento de câncer gastrointestinal e esôfago em adultos, câncer de tireoide e outros distúrbios dessa glândula e metemoglobinemia em bebês de até três meses de idade e em adultos com deficiência enzimática, conhecida como "Síndrome do Bebê Azul"9,10,11.

Vale ainda ressaltar, que níveis elevados de íons de nitrato no ambiente não são um agravante apenas para saúde humana, mas também para o próprio ambiente, visto que o uso exacerbado de adubos nitrogenados no solo pode acarretar o processo de salinização do mesmo, tornando-o impróprio para o desenvolvimento da lavoura $^{12}$. Os altos níveis de íons de nitrato nos cursos d'água também podem culminar no processo de eutrofização, destruindo a fauna e flora local ${ }^{13}$.

O objetivo desse estudo foi avaliar a qualidade água quanto à concentração de íon nitrato em nitrogênio no sistema de distribuição público na área de abrangência da Rede Regional de Saúde (RRAS) 11 - Departamento Regional de
Saúde (DRS) XI do Estado de São Paulo e poços subterrâneos particulares.

\section{METODOLOGIA}

O estudo foi realizado no Centro de Laboratório Regional do Instituto Adolfo Lutz de Presidente Prudente-V (CLR-IAL-PP-V) durante o período de março a junho de 2016. O CLR-IAL-PP$\checkmark$ recebe amostras de Água de abastecimento de 45 municípios da área de abrangência da Rede Regional de Saúde (RRAS) 11 - Departamento Regional de Saúde (DRS) XI do Estado de São Paulo. A Rede Regional de Atenção à Saúde 11 de Presidente Prudente/SP (RRAS 11) é composta por dois Grupos de Vigilância (GVs): o GVXXI de Presidente Prudente da qual subdividem dois Colegiados de Gestão Regional (CGRs): Alta Sorocabana e Alto Capivari e o GVXXII de Presidente Venceslau da qual subdividem três (3) CGRs: Pontal do Paranapanema, Extremo Oeste e Alta Paulista ${ }^{14}$.

Os municípios que compõem o CGR da Alta Paulista são: Dracena, Flora Rica, Irapuru, Junqueirópolis, Monte Castelo, Nova Guataporanga, Ouro Verde, Panorama, Paulicéia, São João do Pau D'alho, Tupi Paulista e Santa Mercedes; Alta Sorocabana: Alfredo Marcondes, Alvares Machado, Anhumas, Caiabu, Emilianópolis, Estrela do Norte, Indiana, Martinópolis, Narandiba, Pirapozinho, Presidente Bernardes, Presidente Prudente, Regente Feijó, Ribeirão dos Índios, Sandovalina, Santo Anastácio, Santo Expedito, Taciba e Tarabai; Alto do Capivari: lepê, João Ramalho, Nantes, Quatá e Rancharia; Extremo Oeste: Caiuá, Marabá Paulista, Piquerobi, Presidente Epitácio, Presidente Venceslau; Pontal do Paranapanema: Euclides da Cunha, Mirante do Paranapanema, Rosana e Teodoro Sampaio.

Foram analisadas 1026 amostras obtidas do Sistema de Abastecimento (provenientes do PROAGUA) dos 45 municípios atendidos e 17 amostras de poços subterrâneos particulares das cidades de Adamantina, Presidente Prudente, Pirapozinho, Osvaldo Cruz, Tupã, Pacaembu, Bataguassu, Álvares Machado e Caiuá.

O método utilizado para a determinação do íon nitrato foi o espectrofotométrico na região do ultravioleta (Spercord S600). A amostra de água foi transferida um balão volumétrico de 100 $\mathrm{mL}$, em seguida foi adicionado $1 \mathrm{~mL}$ de ácido clorídrico 1,0 $\mathrm{M}$ e homogeneizado. Após este procedimento, a absorbância foi lida a $205 \mathrm{~nm}$. Para determinar a quantidade de nitrogênio 
nítrico correspondente, utilizou-se a curvapadrão previamente estabelecida no CLR-IAL-PP$\mathrm{V}^{15}$.

A Portaria no 2914/2011 do Ministério da Saúde $^{5}$ expressa o valor do íon nitrato em nitrogênio, determinando um teor máximo de 10 $\mathrm{mg} / \mathrm{L}$, por isso para expressar em nitrogênio, todos os resultados de concentração de íon nitratos obtidos pela curva analítica préestabelecida foram divididos pelo fator número "4,428", fator que foi obtido através da proporção entre as massas molares de nitrato e nitrogênio, como mostra a seguir: 1 (um) mol de íon nitrato $\left(\mathrm{NO}_{3}{ }^{-}\right) \div 1$ (um) mol de átomo de nitrogênio $(\mathrm{N})=62 \mathrm{~g} \div 14 \mathrm{~g}=4,428$.

\section{RESULTADOS}

Do total de amostras de água de abastecimento, 429 (41,8\%) apresentaram concentração < 1,0 mg/L; 480 (46,8\%) entre 1,0 e $5,0 \mathrm{mg} / \mathrm{L}$ e $117(11,4 \%)$ na faixa entre 6,0 a 10,0 $\mathrm{mg} / \mathrm{L}$ para o íon nitrato em nitrogênio. Nenhuma amostra apresentou concentração maior que 10 $\mathrm{NO}_{3}{ }^{-} \mathrm{N}$ em $\mathrm{mg} / \mathrm{L}$. Já entre as amostras de poços, 5 apresentaram concentração maior que 10 $(29,0 \%) \mathrm{NO}_{3}{ }^{-} \mathrm{N}$ em $\mathrm{mg} / \mathrm{L}$ (Tabela 1$)$. Vale ressaltar que as 5 amostras contaminadas pertenciam ao mesmo endereço, porém pontos de amostragem diferentes.

Tabela 1. Resultado das concentrações de $\mathrm{NO}_{3}{ }^{-} \mathrm{N}$ em $\mathrm{mg} / \mathrm{L}$ em água de abastecimento e poços subterrâneos.

\begin{tabular}{lccccc}
\hline & \multicolumn{5}{c}{ Concentração de Nitrato (NO $\left.\mathbf{N}_{\mathbf{3}} \mathbf{N} \mathbf{~ m g} / \mathbf{L}\right)$} \\
\hline Tipos de amostras & $<1,0$ & 1,0 a 5,0 & 6,0 a 10,0 & $>10,0$ & Total \\
\hline Água de Abastecimento PROAGUA & 429 & 480 & 117 & 0 & 1026 \\
\hline Poços subterrâneos & 4 & 5 & 3 & 5 & 17 \\
\hline
\end{tabular}

Os resultados também foram divididos por região de saúde, onde a região do Alto do Capivari apresentou a maior quantidade de amostras de água de abastecimento na concentração entre 6,0 a 10,0 mg/L, 22 do total de 108 (20,4\%). Em seguida, a região da Alta Paulista com 45 amostras do total de 244
(18,4\%), Pontal do Paranapanema 16 de 120 (13,3\%), Alta Sorocabana com 33 de 438 (7,5\%) e Extremo Oeste Paulista que apresentou 1 amostra nessa faixa do total de $116(0,9 \%)$ (Figura 1).

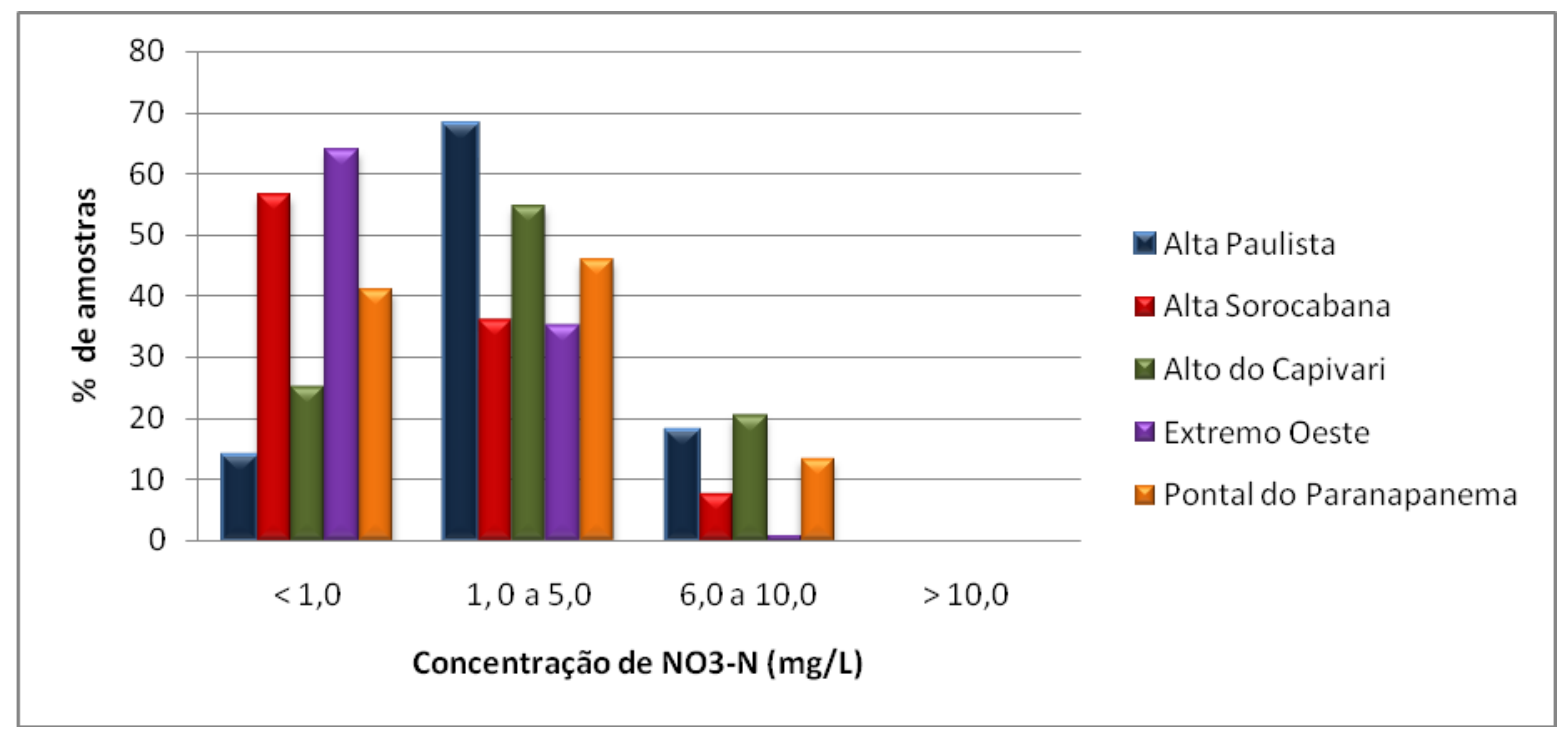

Figura 1. Resultado em porcentagem das concentrações de $\mathrm{NO}_{3}{ }^{-} \mathrm{N}$ em $\mathrm{mg} / \mathrm{L}$ divididos por regiões de saúde em água de abastecimento. 
Dentre as 17 amostras de poços analisadas, $29,0 \%$ apresentaram maior que 10,0
$\mathrm{NO}_{3}{ }^{-} \mathrm{N}$ em $\mathrm{mg} / \mathrm{L}$ e $18,0 \%$ estavam na faixa entre 6,0 a 10,0, como apresentado na Figura 2.

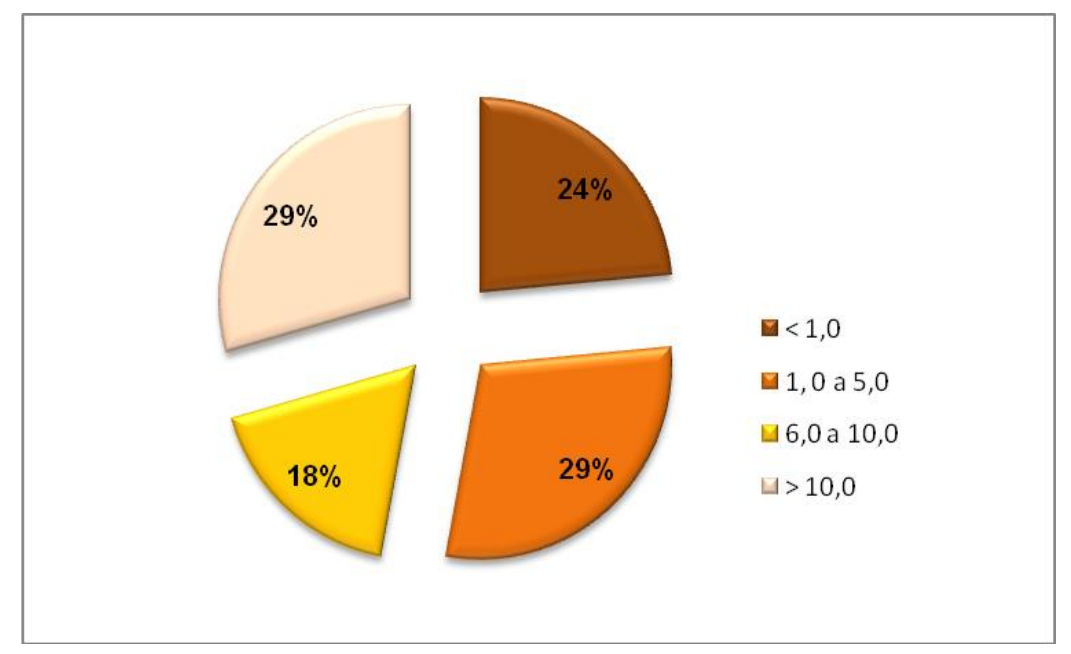

Figura 2. Resultado das amostras de poços subterrâneos divididos em diferentes concentrações de $\mathrm{NO}_{3}{ }^{-} \mathrm{N}$ em $\mathrm{mg} / \mathrm{L}$.

\section{DISCUSSÃO}

Apesar da legislação brasileira ${ }^{5}$ considerar um risco para a saúde o consumo de água contaminada com nitrato em uma concentração acima de 10,0 mg/L, a Companhia Ambiental do Estado de São Paulo (CETESB), que realiza o monitoramento das águas subterrâneas do Estado desde 1977, estabelece que a concentração de $5,0 \mathrm{mg} / \mathrm{L}$ de nitrato seja utilizada como valor de prevenção, servindo para elaboração de ações preventivas e monitoramento da aplicação de resíduos no solo ${ }^{6}$. Os resultados obtidos das águas de abastecimento não apresentaram concentrações maiores que $10,0 \mathrm{mg} / \mathrm{L}$ de íon nitrato em nitrogênio, mas $117(11,4 \%)$ ficaram na faixa de 6,0 a $10,0 \mathrm{mg} / \mathrm{L}$, mostrando a necessidade de uma maior atenção nessas regiões.

Resultados semelhantes foram apresentados por Reis ${ }^{16}$, que no estudo realizado na cidade de Mossoró-RN, as 29 amostras coletadas da rede de abastecimento (torneiras e bebedouros) do Campus da Universidade Estadual do Rio Grande do Norte não apresentaram nitrato com valores superiores a $10,0 \mathrm{mg} / \mathrm{L}$. Em Presidente Prudente-SP, ao ser analisado 5 amostras de afloramentos do Aquífero Bauru, utilizadas para consumo humano, todas estavam adequadas para o íon nitrato ${ }^{17}$. Trindade et al. ${ }^{18}$ ao realizar um estudo em Macapá-AP em três escolas públicas da cidade, analisaram 9 amostras provenientes da caixa d'água, torneira da cozinha e bebedouro e todas amostras apresentaram concentrações que atendem os padrões da legislação.

De todas as amostras de água de abastecimento analisadas, o município de Rosana foi o que mais apresentou altas concentrações de nitrato em nitrogênio. Das 33 analisadas, 5 apresentaram concentrações entre 9 e 10,0 $\mathrm{mg} / \mathrm{L}$. Esses resultados mostram que há uma necessidade de monitoramento neste município, pois as concentrações encontradas estão muito próximas ao permitido pela legislação.

Ao contrário do resultado obtido com as amostras do Sistema de Abastecimento, 5 $(29,0 \%)$ amostras de poços apresentaram concentração de nitrato maior que $10 \mathrm{mg} / \mathrm{L}$. A contaminação de poços por nitrato em nível de propriedade urbana ou rural podem ocorrer pela falta de manutenção do reservatório, local inadequado de instalação, sendo que a maior fonte de contaminação das águas subterrâneas é proveniente de despejos domésticos, industriais e chorume ${ }^{4}$.

De acordo com o estudo realizado por Godoy et al. ${ }^{19}$, a cidade de Presidente PrudenteSP apresentou $28,4 \%$ dos 134 poços pesquisados com concentrações acima do limite aceitável, assim como no presente estudo, onde $5(29,0 \%)$ poços de Presidente Prudente apresentaram concentrações maior que $10 \mathrm{mg} / \mathrm{L}$. 
No estudo realizado na região de Ribeirão Preto-SP entre 2011 e 2012, foi identificada a contaminação de 38 (46,7\%) poços, dos 75 estudados $^{20}$. Em outro estudo realizado na região de Marília-SP, cidade abastecida também pelo Aquífero Bauru, foram analisadas 46 amostras de 21 poços, e 8 (18,0\%) estavam com a concentração de nitrato acima do padrão de potabilidade $^{21}$. Em Presidente Prudente-SP, foi realizado um estudo com amostras de 21 poços da cidade, sendo encontradas concentrações de nitrato acima do permitido em $2(9,0 \%)$ desses poços ${ }^{22}$. Esses três estudos têm em comum a área onde essas amostras apresentaram concentrações de nitrato impróprias, pois são regiões com ocupações urbanas mais antigas da cidade. Estas áreas geralmente apresentavam sistemas de saneamento com fossas negras e sépticas. Além disso, são regiões que pode também ocorrer vazamento das redes coletoras de esgoto ${ }^{19,21}$.

\section{CONCLUSÃO}

O estudo demonstra que, apesar de nenhuma amostra de rede de abastecimento ter apresentado sua concentração maior que o limite permitido, muitas estavam na concentração considerada de prevenção. Portanto o monitoramento sempre se faz necessário, principalmente porque a maior parte da contaminação por nitrato é de atividade antrópica. Esse estudo permite observar a importância da análise do íon nitrato em nitrogênio ser inclusa junto com as outras análises realizadas pelo PROAGUA.

No caso das amostras de poços subterrâneos que apresentaram teores de nitrato acima do permitido, a iniciativa de reduzir essas taxas cabe a cada explorador e a conscientização se faz necessária devido aos problemas que o nitrato pode causar, principalmente se esta água for utilizada para consumo humano.

\section{CONFLITOS DE INTERESSE}

Os autores declaram não haver qualquer potencial conflito de interesse que possa interferir na imparcialidade deste trabalho científico.

\section{REFERÊNCIAS}

1. Gomes, MAF. Água: sem ela seremos o planeta Marte de amanhã. Embrapa Meio Ambiente, mar. 2011. Acesso em: 24 mai. 2016. Disponível em: https://www.infoteca.cnptia.embrapa.br/infotec a/bitstream/doc/921047/1/2011AM02.pdf

2. Daneluz D, Tessaro D. Padrão físico-químico e microbiológico da água de nascentes e poços rasos de propriedades rurais da região sudoeste do Paraná. Arq Inst Biol. 2015;82:1-5. DOI: http://dx.doi.org/10.1590/1808-1657000072013

3. Centro de vigilância sanitária. Água para consumo humano - Proágua. Acesso em: 4 mai. $2016 . \quad$ Disponível em: http://www.cvs.saude.sp.gov.br/prog det.asp?te codigo $=13 \& \mathrm{pr}$ codigo $=13$

4. Biguelini CP, Gumy MP. Saúde ambiental: índices de nitrato em águas subterrâneas de poços profundos na região sudoeste do Paraná. Rev Faz Ciência. 2012;14 (20):153-75.

5. Brasil. Portaria no 2914, de 12 de dez. 2011 do Ministério da Saúde. Estabelece os procedimentos e responsabilidades relativos ao controle e vigilância da qualidade da água para consumo humano e seu padrão de potabilidade, e dá outras providências. Diário Oficial da União, Brasília, DF, 14 de dez. 2011.

6. Cetesb. Qualidade das águas subterrâneas do estado de São Paulo 2013-2015 [recurso eletrônico] / CETESB ; Execução Rosângela Pacini Modesto... [et al.].; Colaboração Blas Marçal Sanchez...[et al.]. - - São Paulo : CETESB, 2016.

7. Neira DF, Terra VT, Prate-Santos R, Barbiéri RS. Impactos do necrochorume nas águas subterrâneas do cemitério de Santa Inês, Espírito Santo, Brasil. Brasil Natureza On-line. 2008;6(1):36-41.

8. Guerreiro RS, Sá MS, Rodrigues, LAP. Avaliação do teor de nitrito e nitrato em alimentos cárneos comercializados em Salvador. Rev Intertox Toxicol Risco Ambiental Soc. 2012;5(1):77-91.

9. Fernícola NG, Gándara de, Azevedo FA. Metemoglobinemia e nitrato nas águas. Rev Saúde Púb. 1981;15(2):242-8. DOI: https://dx.doi.org/10.1590/S003489101981000200009

10. Who/Unicef. Progress on sanitation and drinking water - 2015. Update and MDG Assessment. Geneva, World Health Organization, 2015.

11. Ward MR, Kilfoy BA, Weyer PJ, Anderson, KE, Folsom, AR, Cerhan JR. Nitrate intake and the risk of thyroid cancer and thyroid disease. Epidemiology. 2010;21(3):389-95 https://dx.doi.org/10.1097/EDE.0b013e3181d620 $\underline{1 \mathrm{~d}}$

12. Medeiros PRF, Silva EFF, Duarte SN, Santos NA, Dantas RML. Determinação do íon nitrato e 
ph no tomateiro sob níveis de salinidade do solo. Fortaleza/CE: IV Winotec, 2012. Acesso em: 5 mai. 2016. Disponível em: http://www.inovagri.org.br/meeting2012/wpcontent/uploads/2012/06/Protocolo259.pdf 13. Amabis JM, Martho GR. Fundamentos da biologia moderna. 3.ed. São Paulo: Editora Moderna; 2003.

14. São Paulo. Secretaria de Estado da Saúde. Rede Regional de Atenção à Saúde 11 Presidente Prudente. Nov, 2012.

15. Instituto Adolfo Lutz. Métodos químicos e físicos para análise de alimentos 4a ed. São Paulo: IMESP; 2004. p. 343-4.

1. 16. Reis SM, Pinto $\mathrm{CHC}$, Rodrigues $\mathrm{BC}$, Benevides MG. Investigação dos íons nitrato e nitrito nas águas de abastecimento do campus central e epílogo de Campus - UERN no município de Mossoró-RN; 2009.

17. Siste NA, Souza, AT. A qualidade da água das minas utilizadas para fins de potabilidade em pontos de afloramentos do aquífero bauru no entorno de Presidente Prudente/SP. Rev Coll Exactarum. 2013;5:141-8. DOI: http://dx.doi.org/.5747/ce.2013.v05.nesp.00006 4

18. Trindade GA, Sá-Oliveira JC, Silva ES. Avaliação da qualidade da água em três Escolas Públicas da Cidade de Macapá, Amapá. Biota Amazônia. 2015;5(1):116-22. DOI: http://dx.doi.org/10.18561/2179-5746

2. 19. Godoy MCTF, Boin MN, Sanaiotti DC, Silva JB. Contaminação das águas subterrâneas por nitrato em Presidente Prudente - SP, Brasil. Rev Instit Adolfo Lutz. 2004;63(2):208-14.

20. Dovidauskas S, Okada IA, Souza, JÁ, Jesus MA, Novas $H$, Rossato RA. A interação entre Vigilância Sanitária e Laboratório de Saúde Pública na detecção da contaminação por nitrato. Rev Vig Sanit. Debate em água subterrânea. 2015;3(1):97-104. DOI: http://dx.doi.org/10.3395/2317-269x.00259

21. Vanier C, Iritani MA, Viotti M, Oda GH, Ferreira LMR. Nitrato nas águas subterrâneas do Sistema Aquífero Bauru, área urbana do município de Marília (SP). Rev Instit Geológico. 2010;31(1/2):1-21. DOI: https://doi.org/10.5935/0100-929X.20100001

22. Santana AT, Vicentini CF, Cuba RMF. Avaliação da presença de nitrato e coliformes em águas subterrâneas de Presidente Prudente - SP. VIII Fórum Ambiental da Alta Paulista. 2012; 8(12): 187-95. Disponível em: http://www.abq.org.br/cbq/2009/trabalhos/5/5244-5918.htm

Recebido para publicação em 05/08/2016

Revisado em 10/08/2016

Aceito em 23/08/2016 